\title{
No Effect of Genome-Wide Significant Schizophrenia Risk Variation at the DRD2 Locus on the Allelic Expression of DRD2 in Postmortem Striatum
}

\author{
Carolina C. Toste ${ }^{a}$ Rodrigo R.R. Duarte ${ }^{b}$ Aaron R. Jeffries ${ }^{c}$ \\ Sashika Selvackadunco ${ }^{d}$ Claire Troakes $^{d}$ Michael C. O'Donovan ${ }^{a}$ \\ Matthew J. Hilla Nicholas J. Bray ${ }^{a}$ \\ ${ }^{a}$ MRC Centre for Neuropsychiatric Genetics and Genomics, Division of Psychological Medicine and Clinical

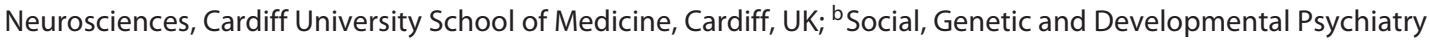 \\ Centre, Institute of Psychiatry, Psychology and Neuroscience, King's College London, London, UK; \\ 'University of Exeter Medical School, Exeter, UK; ${ }^{\mathrm{d}}$ Department of Basic and Clinical Neuroscience, Institute of \\ Psychiatry, Psychology and Neuroscience, King's College London, London, UK
}

\section{Keywords}

Dopamine D2 receptor · DRD2 · Schizophrenia - Genetics · Gene expression

\begin{abstract}
A genome-wide significant association has been reported between non-coding variants at the dopamine D2 receptor (DRD2) gene locus and schizophrenia. However, effects of identified schizophrenia risk alleles on DRD2 function are yet to be demonstrated. Using highly sensitive measures of allele-specific expression, we have assessed cis-regulatory effects associated with genotype at lead SNP rs2514218 on $D R D 2$ expression in the adult human striatum. No significant differences were observed in the extent of allelic expression imbalance between samples that were genomic heterozygotes for rs 2514218 (where cis-regulatory effects of the risk allele are compared with those of the non-risk allele within individual subjects) and samples that were homozygous for rs 2514218 (where cis-regulatory effects of this SNP on each
\end{abstract}

expressed $D R D 2$ allele will be equal). We therefore conclude that rs2514218 genotype is not associated with large effects on overall DRD2 RNA expression, at least in postmortem adult striatum. Alternative explanations for the genetic association between this variant and schizophrenia include effects on $D R D 2$ that are transcript specific, restricted to minor $D R D 2$-expressing cell populations or elicited only under certain physiological circumstances, or mediation through effects on another gene (or genes) at the locus.

(C) 2019 The Author(s)

Published by S. Karger AG, Basel

\section{Introduction}

The dopamine D2 receptor is a primary therapeutic target of all current antipsychotic drugs [1]. It is encoded by a single gene, $D R D 2$, which gives rise to two major receptor isoforms (D2-long and D2-short) through alternative RNA splicing [2]. An early focus of candidate gene association studies (e.g., $[3,4]), D R D 2$ has been the sub-

\begin{tabular}{ll}
\hline KARGER & $\begin{array}{l}\text { () } 2019 \text { The Author(s) } \\
\text { Published by S. Karger AG, Basel }\end{array}$ \\
E-Mail karger@karger.com & $\begin{array}{l}\text { This article is licensed under the Creative Commons Attribution 4.0 } \\
\text { International License (CC BY) (http://www.karger.com/Services/ } \\
\text { OpenAccessLicense). Usage, derivative works and distribution are } \\
\text { permitted provided that proper credit is given to the author and the } \\
\text { original publisher. }\end{array}$
\end{tabular}


ject of renewed interest as a potential schizophrenia susceptibility gene following observation of genome-wide significant association between schizophrenia and variants at the DRD2 locus in a large genome-wide association study (GWAS) [5]. The single nucleotide polymorphism (SNP) at the locus exhibiting most significant association with schizophrenia in that study was rs 2514218 $\left(p=2.75 \times 10^{-11}\right.$ in the entire sample), a non-coding variant located $47 \mathrm{~kb}$ upstream of the most $5^{\prime}$ known DRD2 exon. This association has been further substantiated in a recent, enlarged GWAS of schizophrenia [6], where finemapping has indicated that rs2514218 is the SNP at the locus with the highest posterior probability $(0.69)$ of being the causal variant.

The schizophrenia risk (C-) allele of rs2514218 is reported to be associated with impaired striatal function [7] and antipsychotic treatment response $[8,9]$. Although these findings are consistent with effects on subcortical dopamine function, the effect on DRD2 associated with rs2514218 genotype is currently unclear. Given its location in non-coding sequence, we hypothesized that rs2514218, or other variants in linkage disequilibrium with it, alter the expression of DRD2 in the human brain. In order to test this hypothesis, we used highly sensitive measures of allele-specific expression to assess effects of rs2514218 genotype on the cis-regulation of DRD2 in the adult striatum, a region where $D R D 2$ is prominently expressed, and where $\mathrm{D} 2$ receptor occupancy predicts the therapeutic response to antipsychotic drugs [10].

\section{Materials and Methods}

Brain Samples

Frozen caudate tissue was obtained postmortem from 120 unrelated adults from the London Neurodegenerative Diseases Brain Bank (UK). All subjects were free from psychiatric or neurological diagnosis at the time of death. Genomic DNA was extracted from all samples using standard phenol/chloroform procedures. Total RNA was extracted using Tri-Reagent (Thermo Fisher Scientific), according to manufacturer instructions. RNA samples were treated with TURBO DNase (Thermo Fisher Scientific) prior to reverse transcription and did not yield a PCR product in the absence of a reverse transcription step. Approximately $1 \mu \mathrm{g}$ of total RNA was reverse transcribed with SuperScript III and random decamers (Thermo Fisher Scientific). Resulting cDNA was diluted 1:7 prior to use.

\section{Genotyping}

Samples were genotyped for schizophrenia risk SNP rs2514218 and DRD2 exonic SNPs rs6277 and rs7116768 (used for measures of $D R D 2$ allele-specific expression) by single base primer extension using SNaPshot ${ }^{\circledR}$ chemistry (Thermo Fisher Scientific) and PCR-amplified genomic DNA. Primer sequences are provided in online supplementary Table S1 (for all online suppl. material, see www.karger.com/doi/10.1159/000501022). There was no significant $(p<0.05)$ deviation from Hardy-Weinberg equilibrium in the genotype distribution of any variant. Linkage disequilibrium $\left(\mathrm{D}^{\prime}\right)$ between genotyped SNPs in the 120 samples was calculated using Haploview version 4.2 [11].

\section{Measurement of DRD2 Allele-Specific Expression}

Measures of allele-specific expression typically make use of SNPs in exonic (i.e., expressed) sequence as endogenous tags to discriminate and relatively quantify the RNA transcribed from each chromosomal copy of a gene in heterozygous subjects. Departure from the expected 1:1 ratio of allelic expression in any given sample indicates heterozygous cis-regulatory influences operating on that gene [12]. A major advantage of this "within-subjects" approach is that it measures variable cis-effects on gene expression while controlling for confounding influences (e.g., trans-effects of other genes, environmental exposures, and tissue variables such as RNA quality), which are expected to impact on both gene copies equally.

Samples identified as genomic heterozygotes for SNPs rs6277, located in the penultimate exon of most protein-coding DRD2 RNA transcripts, and SNP rs7116768, located in a $5^{\prime}$ exon utilised by a smaller subset of $D R D 2$ transcripts, were used to measure $D R D 2$ allelic expression. Known demographics for these samples are provided in online supplementary Table S2. The position of these SNPs in relation to Ensembl transcripts is shown in Figure 1. Sequence containing rs6277 or rs7116768 was PCR-amplified from cDNA alongside genomic DNA from the same heterozygous subjects using primers based on single exon sequence (online suppl. Table S1). PCR products were treated with shrimp alkaline phosphatase and exonuclease I (New England Biolabs) to inactivate nucleotides and primers for downstream steps. Alleles of each SNP were discriminated and relatively quantified by SNaPshot ${ }^{\circledR}$ primer extension (Thermo Fisher Scientific), using extension primers detailed in online supplementary Table S1. Reaction products were electrophoresed on an Applied Biosystems 3130xl Genetic Analyzer and peak heights of allele-specific extended primers were determined using GeneMarker software (SoftGenetics). Peak heights, representing the relative abundance of each allele, were used to calculate an allele ratio for each reaction. The average allele ratio from all genomic DNA samples on each plate was used as a correction factor for all genomic DNA and cDNA allele ratios, since this can be assumed to reflect a perfect 1:1 ratio of the two alleles and can therefore be used to correct for any inequalities in allelic representation specific to the assay [12]. Four technical replicates were performed for each cDNA sample, alongside $\mathrm{H}_{2} \mathrm{O}$ as a negative control. Any samples showing poor reproducibility in cDNA allele ratios (standard deviation/mean $>0.25$ ) were excluded from further analyses.

\section{Testing Effect of rs2514218 Genotype on DRD2 Allelic}

Expression

$\mathrm{D}^{\prime}$ between rs6277 and rs2514218 was 0.51 in our samples, and 0.65 between rs7116768 and rs2514218. This modest level of linkage disequilibrium between the SNPs used to assay DRD2 allelespecific expression and the schizophrenia risk SNP means that, for any given sample, the phase between expressed DRD2 alleles and the risk allele is uncertain. We therefore assessed potential cis-regulatory effects on DRD2 associated with the rs2514218 risk allele 


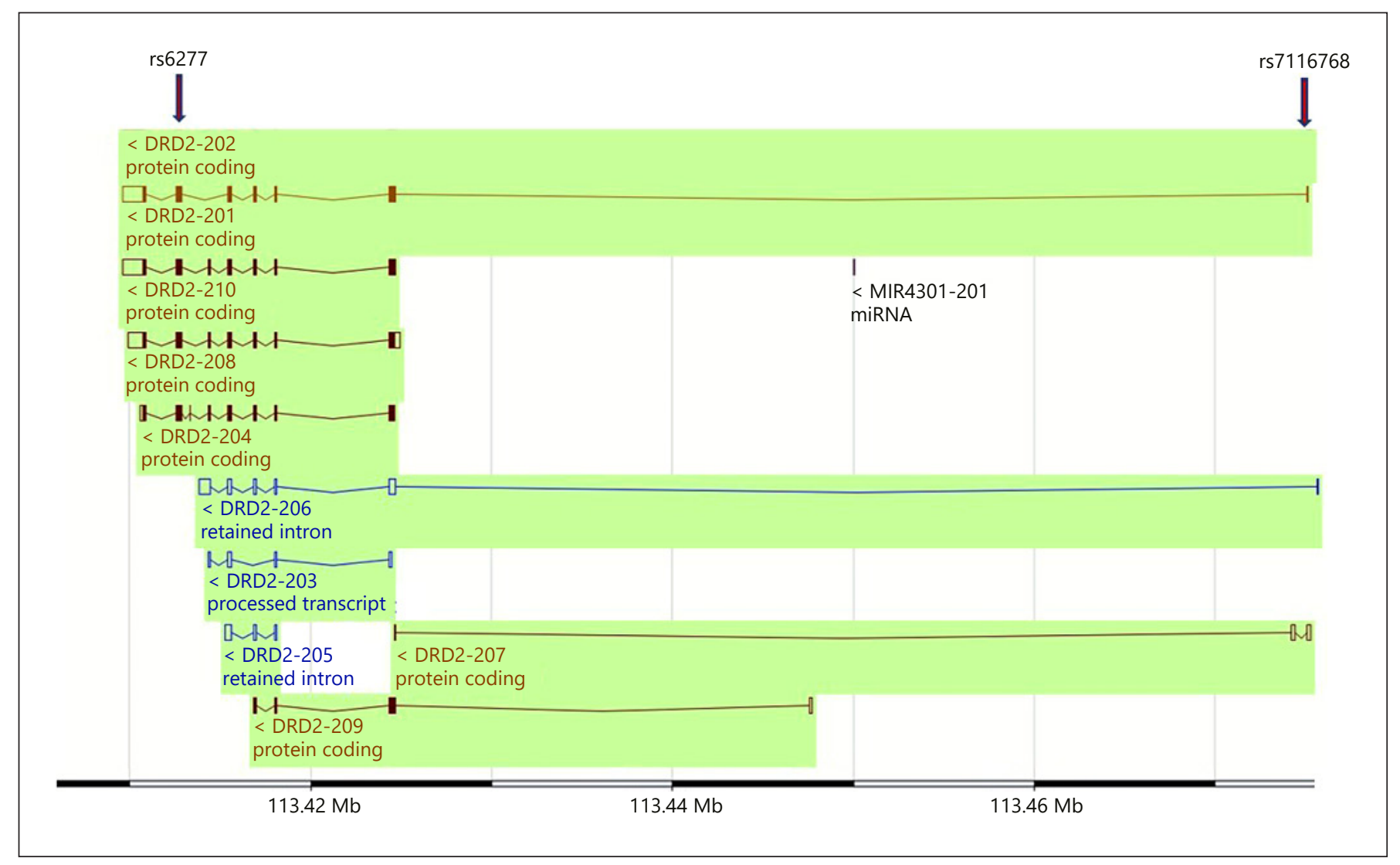

Fig. 1. Position of expressed single nucleotide polymorphisms rs6277 and rs7116768, used to assay DRD2 allelespecific expression in this study, in relation to DRD2 transcripts annotated by Ensembl (release 95). Location in $\mathrm{Mb}$ on chromosome 11 is shown according to Genome Reference Consortium Human Build 38. Schizophrenia risk SNP rs2514218 is located at $113.52 \mathrm{Mb}$.

by comparing the mean deviation from the average (1:1) genomic allele ratio observed in rs2514218 heterozygotes (where any cisregulatory effects of the risk allele are compared with those from the non-risk allele) and rs2514218 homozygotes (where cis-regulatory effects of rs2514218 alleles are equal). Comparisons were performed by two-tailed $t$ tests using SPSS 25.0 software. There was no significant difference in the variance of allelic expression imbalance between rs2514218 heterozygotes and homozygotes at either expressed SNP (Levene's test $p>0.05$ ).

\section{Results}

Allele-specific expression at DRD2 SNP rs6277 was assessed in 39 heterozygous samples. Of these, 18 were additionally heterozygous for schizophrenia risk SNP rs2514218, while 21 were homozygous. Figure 2 shows allelic expression ratios in cDNA from rs2514218 homozygotes and heterozygotes, alongside allelic ratios derived from genomic DNA from the same subjects. Two samples that were heterozygous for schizophrenia risk SNP rs2514218 exhibited pronounced allelic expression imbalance, with increased relative expression of the rs6277 A-allele by 35 and 40\%. However, the mean deviation from the expected 1:1 ratio of the two alleles was similar for rs2514218 homozygotes, at 10\%, and heterozygotes, at $12 \%(p=0.46)$.

SNP rs7116768 is located within the most $5^{\prime}$ DRD2 exon, and the closest known exon to schizophrenia risk SNP rs2514218. This exon is predicted to be contained within two Ensembl DRD2 transcripts, including DRD2201 (ENST00000346454.7), encoding D2-short. Allelespecific expression at rs7116768 was assessed in 23 heterozygous samples. Of these, 7 were additionally heterozygous for schizophrenia risk SNP rs2514218 and 16 were homozygous. With the exception of one rs251421 heterozygote, which exhibited an allelic expression imbalance of 


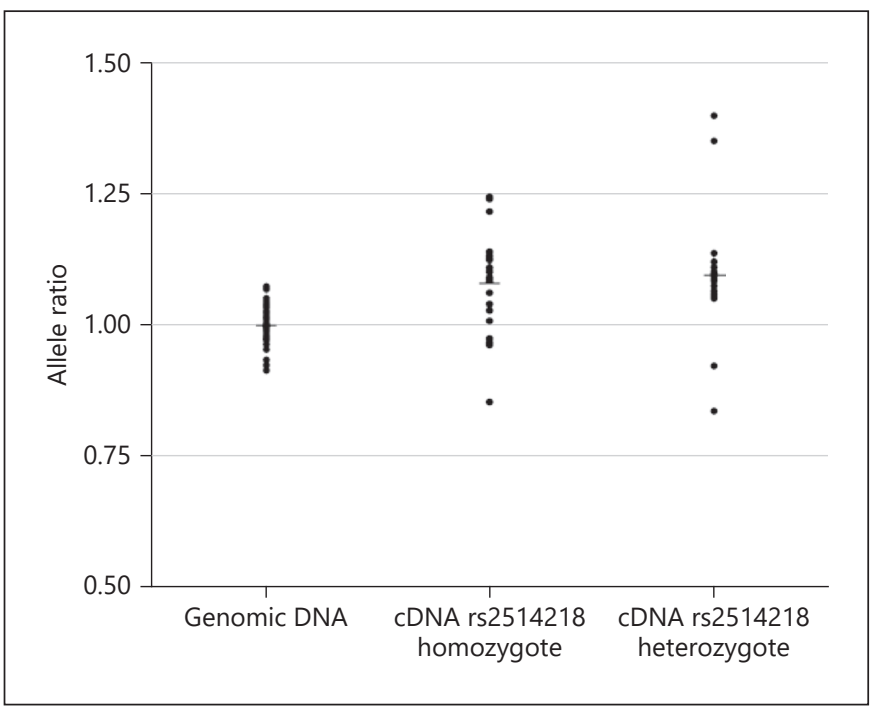

Fig. 2. Allele ratios at expressed DRD2 SNP rs6277 in the caudate of adult subjects who are either homozygous or heterozygous for schizophrenia risk SNP rs2514218. Data points represent the average allele ratio in genomic DNA or cDNA from each sample. Mean allele ratios are indicated by horizontal lines. There is no significant difference in the extent of allelic expression imbalance (departure from the average 1:1 genomic ratio) between cDNA from rs2514218 homozygotes and rs2514218 heterozygotes $(p=0.46)$.

$18 \%$, all samples displayed cDNA allele ratios close to the 1:1 genomic average, with a mean deviation of $5 \%$ in rs 251421 heterozygotes and $2 \%$ in rs251421 homozygotes $(p=0.56)$ (Fig. 3).

\section{Discussion}

We have used highly sensitive measures of allele-specific expression to assess cis-regulatory effects associated with rs2514218 genotype on DRD2 expression in the adult human striatum. Although a small number of rs2514218 heterozygotes displayed pronounced allelic expression imbalance, the majority exhibited cDNA allele ratios similar to those observed in rs2514218 homozygotes, where cis-regulatory effects of this SNP on each expressed DRD2 allele will be equal. We therefore conclude that rs 2514218 genotype is not associated with consistent effects on overall DRD2 RNA expression, at least in postmortem adult striatum.

The allele-specific expression method is considerably more powerful than standard expression quantitative trait loci (eQTL) approaches for detecting cis-regulatory variation because it controls potential trans-acting con-

No Effect of rs2514218 Genotype on

DRD2 Allelic Expression

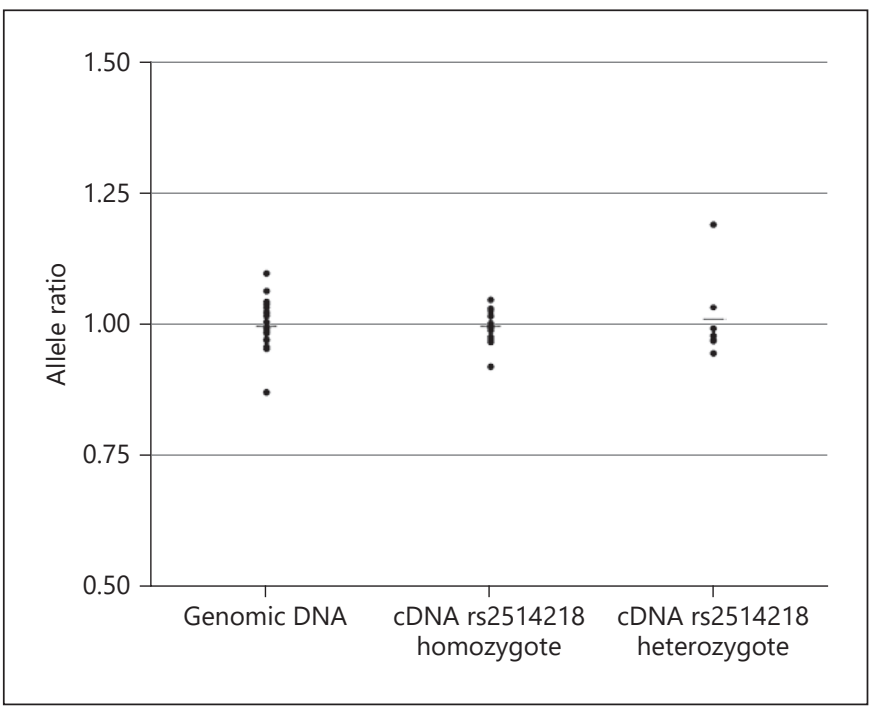

Fig. 3. Allele ratios at expressed DRD2 SNP rs7116768 in the caudate of adult subjects who are either homozygous or heterozygous for schizophrenia risk SNP rs2514218. Data points represent the average allele ratio in genomic DNA or cDNA from each sample. Mean allele ratios are indicated by horizontal lines. There is no significant difference in the extent of allelic expression imbalance (departure from the average 1:1 genomic ratio) between cDNA from rs2514218 homozygotes and rs2514218 heterozygotes $(p=0.56)$.

founders that will act on both chromosomal copies of an expressed gene to the same extent (i.e., affecting total, but not allele-specific, expression). Indeed, eQTL approaches have been found to require up to 8 times more samples than allele-specific expression measures to detect the effects of the same cis-regulatory variants [13]. However, in finding no evidence for an association between rs 2514218 genotype and DRD2 expression, our data are broadly consistent with publicly available eQTL data generated from postmortem human brain tissue. Thus, in the BRAINEAC database (available at http://www.braineac.org/) [14], which includes 10 adult brain regions, genotype at rs 2514218 is associated with more significant effects on the expression of several other genes at the DRD2 locus (e.g., REXO2, HTR3A, NCAM1, TTC12, and ANKK1) than on $D R D 2$ itself, although in no case is the evidence strong (smallest $p=0.00087$ [uncorrected] for REXO2 expression in putamen). Similarly, rs2514218 is not associated with significant effects on the expression of any gene in any of the 13 adult brain regions included in the GTEx version 7 release (data available at https://gtexportal.org/ home/) [15], although significant (but opposing) effects of the risk allele on DRD2 expression are observed in esophagus and skeletal muscle. SNP rs2514218 is not as- 
sociated with significant effects on the expression of any gene in the adult dorsolateral prefrontal cortex in the BrainSeq phase 1 data from the Lieber Institute (data available at http://eqtl.brainseq.org/phase1/) [16], while the recent large-scale eQTL mapping study performed by the PsychENCODE Consortium in adult prefrontal cortex (data available at http://resource.psychencode.org/) [17] identified no variants associated with altered $D R D 2$ expression at a false discovery rate $(\mathrm{FDR})<0.05$. In addition, we found no evidence that rs 2514218 or any other common variants are eQTL (at FDR <0.05) for DRD2 in second-trimester human fetal brain [18], despite detection of $D R D 2$ expression at this early stage of development (data available at https://doi.org/10.6084/m9.figshare.6881825).

Given the current lack of evidence for an effect of rs2514218 genotype on DRD2 expression in postmortem brain, alternative explanations for the highly significant association between this SNP and schizophrenia are required. As we used expressed SNPs common to multiple DRD2 mRNA transcripts to assay allele-specific expression, it is possible that effects of rs2514218 genotype are restricted to specific transcripts that constitute only a fraction of the DRD2 expression measured. It is also possible that effects on $D R D 2$ are specific to a minority cell population within the striatum (or other brain region), or that these effects are elicited only in circumstances of neural activity that are not captured in postmortem tissue. These possibilities may be addressed in future studies through RNA sequencing (providing transcript-specific measures), single cell analyses, and in vitro assessments. However, while $D R D 2$ is a prime functional candidate for schizophrenia, a fourth possibility - that susceptibility is mediated through a different gene (or genes) at the locus - should not be discounted.

\section{Acknowledgements}

Adult human brain tissue was supplied by The London Neurodegenerative Diseases Brain Bank, which receives funding from the Medical Research Council (UK) and, as part of the Brains for Dementia Research programme, is jointly funded by Alzheimer's Research UK and Alzheimer's Society.

\section{Statement of Ethics}

Ethical approval for this study was provided by The Joint South London and Maudsley and The Institute of Psychiatry NHS Research Ethics Committee (REF: PNM/12/13-102). Subjects were approached in life for written consent for brain banking, and all tissue donations were collected and stored following legal and ethical guidelines.

\section{Disclosure Statement}

The authors have no conflicts of interest to declare.

\section{Funding Sources}

This work was supported by a Medical Research Council (UK) project grant to N.J.B. (G0802166), a Fundacao AstraZeneca (FAZ) Innovate Competition grant to C.C.T., a grant from the Coordination for Improvement of Higher Education Personnel (CAPES, Brazil, BEX 1279/13-0) to R.R.R.D., and a Medical Research Council (UK) Centre grant (MR/L010305/1).

\section{Author Contributions}

C.C.T. and N.J.B. designed the study. Laboratory work was carried out by C.C.T., R.R.R.D., A.R.J., S.S., and C.T. Data were analysed by C.C.T. and N.J.B. and interpreted by C.C.T., M.J.H., M.C.O'D., and N.J.B. The manuscript was written by N.J.B. with input and approval from all other authors.

\section{References}

1 Seeman P. Targeting the dopamine D2 receptor in schizophrenia. Expert Opin Ther Targets. 2006 Aug;10(4):515-31.

2 Dal Toso R, Sommer B, Ewert M, Herb A, Pritchett DB, Bach A, et al. The dopamine D2 receptor: two molecular forms generated by alternative splicing. EMBO J. 1989 Dec;8(13): 4025-34.

3 Laurent C, Bodeau-Péan S, Campion D, d'Amato T, Jay M, Dollfus S, et al. No major role for the dopamine D2 receptor Ser$[\{\mathrm{GT}\}]$ Cys311 mutation in schizophrenia. Psychiatr Genet. 1994;4(4):229-30.
4 Arinami T, Gao M, Hamaguchi H, Toru M. A functional polymorphism in the promoter region of the dopamine $\mathrm{D} 2$ receptor gene is associated with schizophrenia. Hum Mol Genet. 1997 Apr;6(4):577-82.

5 Schizophrenia Working Group of the Psychiatric Genomics Consortium. Biological insights from 108 schizophrenia-associated genetic loci. Nature. 2014 Jul;511(7510):421-7.
6 Pardiñas AF, Holmans P, Pocklington AJ, Escott-Price V, Ripke S, Carrera N, et al.; GERAD1 Consortium; CRESTAR Consortium. Common schizophrenia alleles are enriched in mutation-intolerant genes and in regions under strong background selection. Nat Genet. 2018 Mar;50(3):381-9.

7 Vink M, de Leeuw M, Luykx JJ, van Eijk KR, van den Munkhof HE, van Buuren M, et al DRD2 schizophrenia-risk allele is associated with impaired striatal functioning in unaffected siblings of schizophrenia patients. Schizophr Bull. 2016 May;42(3):843-50. 
8 Huang E, Maciukiewicz M, Zai CC, Tiwari AK, Li J, Potkin SG, et al. Preliminary evidence for association of genome-wide significant DRD2 schizophrenia risk variant with clozapine response. Pharmacogenomics. 2016;17(2):103-9.

9 Zhang JP, Robinson DG, Gallego JA, John M, $\mathrm{Yu}$ J, Addington J, et al. Association of a schizophrenia risk variant at the DRD2 locus with antipsychotic treatment response in first-episode psychosis. Schizophr Bull. 2015 Nov;41(6):1248-55.

10 Agid O, Mamo D, Ginovart N, Vitcu I, Wilson AA, Zipursky RB, et al. Striatal vs extrastriatal dopamine D2 receptors in antipsychotic response-a double-blind PET study in schizophrenia. Neuropsychopharmacology. 2007 Jun;32(6):1209-15.

11 Barrett JC, Fry B, Maller J, Daly MJ. Haploview: analysis and visualization of $L D$ and haplotype maps. Bioinformatics. 2005 Jan; 21(2):263-5.
12 Bray NJ, Buckland PR, Owen MJ, O'Donovan MC. Cis-acting variation in the expression of a high proportion of genes in human brain. Hum Genet. 2003 Jul;113(2):149-53.

13 Almlöf JC, Lundmark P, Lundmark A, Ge B, Maouche S, Göring HH, et al. Powerful identification of cis-regulatory SNPs in human primary monocytes using allele-specific gene expression. PLoS One. 2012;7(12):e52260.

14 Ramasamy A, Trabzuni D, Guelfi S, Varghese V, Smith C, Walker R, et al.; UK Brain Expression Consortium; North American Brain Expression Consortium. Genetic variability in the regulation of gene expression in ten regions of the human brain. Nat Neurosci. 2014 Oct;17(10):1418-28.
15 Battle A, Brown CD, Engelhardt BE, Montgomery SBGTEx ConsortiumLaboratory, Data Analysis \&Coordinating Center (LDACC) - Analysis Working Group, et al.; eQTL manuscript working group. Genetic effects on gene expression across human tissues. $\mathrm{Na}$ ture. 2017 Oct;550(7675):204-13.

16 Jaffe AE, Straub RE, Shin JH, Tao R, Gao Y, Collado-Torres L, et al.; BrainSeq Consortium. Developmental and genetic regulation of the human cortex transcriptome illuminate schizophrenia pathogenesis. Nat Neurosci. 2018 Aug;21(8):1117-25.

17 Wang D, Liu S, Warrell J, Won H, Shi X, Navarro FC, et al.; PsychENCODE Consortium. Comprehensive functional genomic resource and integrative model for the human brain. Science. 2018 Dec;362(6420):eaat8464.

18 O'Brien HE, Hannon E, Hill MJ, Toste CC Robertson MJ, Morgan JE, et al. Expression quantitative trait loci in the developing human brain and their enrichment in neuropsychiatric disorders. Genome Biol. 2018 Nov; 19(1):194. 